\title{
Efeitos da Musicoterapia aplicada às crianças com condições complexas crônicas
}

\author{
Effects of Music Therapy applied to children with complex chronic health conditions \\ Efectos de la Musicoterapia aplicada a niños con condiciones crónicas complejas
}

Recebido: 17/01/2022 | Revisado: 21/01/2022 | Aceito: 26/01/2022 | Publicado: 28/01/2022

\author{
Renata de Moura Bubadué \\ ORCID: https://orcid.org/0000-0001-8121-1069 \\ Faculdade de Ciências e Educação Sena Aires, Brasil \\ E-mail: renatabubadue@gmail.com \\ Fabiano Pereira dos Reis \\ ORCID: https://orcid.org/0000-0002-2107-6659 \\ Faculdade de Ciências e Educação Sena Aires, Brasil \\ E-mail: fabianoreis.enfermagem@gmail.com \\ Francisco das Chagas dos Santos da Silva \\ ORCID: https://orcid.org/0000-0002-6552-3764 \\ Faculdade de Ciências e Educação Sena Aires, Brasil \\ E-mail: professorchagas@gmail.com \\ Érika Eberlline Pacheco dos Santos \\ ORCID: https://orcid.org/0000-0002-2130-4228 \\ Universidade Federal de Santa Maria, Brasil \\ E-mail: erikaeberlline@live.com \\ Marcelo Ribeiro Primeira \\ ORCID: https://orcid.org/0000-0001-9735-6502 \\ Universidade Federal de Santa Maria, Brasil \\ E-mail: mrp_sm@hotmail.com
}

\begin{abstract}
Resumo
Este artigo tem como objetivo descrever os efeitos da musicoterapia aplicada a crianças com condições crônicas complexas sob a ótica do familiar. Pesquisa interventiva e participativa realizada com onze crianças com condições crônicas complexas de saúde no período de março a abril de 2021 em uma cidade de médio porte do centro-oeste do Brasil. A pesquisa foi aprovada pelo Comitê de Ética sob o CAAE 41238820.1.0000.5595. Segundo os familiares, houve mudança de comportamento durante e após as sessões de musicoterapia. A musicoterapia melhorou a comunicação, o foco, a interação, o relaxamento e o vínculo das crianças com os profissionais de saúde e familiares.

Palavras-chave: Musicoterapia; Enfermagem; Criança; Enfermagem pediátrica.
\end{abstract}

\begin{abstract}
This article aims to describe the effects of music therapy applied to children with complex chronic health conditions under the family member's optics. Interventional and participatory research carried with eleven children with complex chronic health conditions from March to April 2021 in a medium-size city in the center-west of Brazil. Research was approved by the Research Ethics Committee under the protocol CAAE 41238820.1.0000.5595. According to the family members, there was a change of behavior during and after the music therapy sessions. Music therapy improved children's communication, focus, interaction, relaxation and bond with the health professionals and family members.

Keywords: Music therapy; Nursing; Child; Pediatric nursing.

\section{Resumen}

Este artículo tiene como objetivo describir los efectos de la musicoterapia aplicada a niños con condiciones crónicas complejas desde la perspectiva de la familia. Investigación intervencionista y participativa realizada con once niños con condiciones de salud crónicas complejas de marzo a abril de 2021 en una ciudad de tamaño medio en el centrooeste de Brasil. La investigación fue aprobada por el Comité de Ética bajo el CAAE 41238820.1.0000.5595. Según los familiares, hubo un cambio de comportamiento durante y después de las sesiones de musicoterapia. La musicoterapia mejoró la comunicación, el enfoque, la interacción, la relajación y el vínculo de los niños con los profesionales de la salud y los miembros de la familia.
\end{abstract}

Palabras clave: Musicoterapia; Enfermería; Niño; Enfermería pediátrica.

\section{Introdução}

Crianças com condições crônicas complexas (CCC) representam um grupo populacional heterogêneo, cujas demandas de cuidados comprometem severamente um ou mais sistemas orgânicos por, ao menos, 12 meses. Essas crianças necessitam de 
algum período de hospitalização e exigem cuidados especializados e contínuos dos serviços de saúde (Moreira et al., 2017).

No Brasil, a terminologia de CCC é utilizada como uma subclassificação das crianças com necessidades especiais de saúde (CRIANES), cuja denominação está relacionada ao grau de dependência que fazem de serviços de saúde. Assim, a definição das crianças com CCC incorpora a presença de demandas de cuidados medicamentosa, dietéticas, de reabilitação física, de linguagem e deglutição prestados por uma equipe multiprofissional por mais de 12 meses (Moreira et al., 2017).

Dentro da população de crianças com CCC, tem-se as crianças com deficiência. Estima-se que 93 a 150 milhões de crianças vivem com algum tipo de deficiência, sendo que 3.43 milhões delas residem no Brasil e tem idades de zero a 14 anos (Unicef, 2013, Brasil, 2010). No Brasil, um estudo aferiu a incidência de hospitalizações delas no ano de 2013 a partir de dados do Sistema de Informações Hospitalares do Sistema Único de Saúde (SUS). Os resultados apontaram a ocorrência de 331 hospitalizações para cada 100 mil crianças. Já o total de internações para essa população nesse mesmo período foi em média de 240 mil (Moura et al, 2017).

Mesmo antes da pandemia da Covid-19, essas crianças experienciavam estresse, ansiedade e medo associados à sua condição de saúde (Graham et al., 2016, Novak \& Honan, 2019, Elmacı \& Cevizci, 2015). Esses sentimentos foram agravados com o isolamento social, tendo sido descritos sob a perspectiva dos familiares cuidadores e dos profissionais de saúde (Clemens et al., 2020, Banerjee et al., 2021, Fegert et al., 2020).

De orientação epistemológica de natureza híbrida, a musicoterapia valoriza elementos da arte e saúde em sintonia. O movimento, a linguagem e o som se entrelaçam em processos de aprendizagem, construção e desenvolvimento psicossocial e cognitivo, fundamentais para a promoção, manutenção e recuperação de saúde. Tem sido descrita como uma alternativa que congrega ciência e arte na promoção da reabilitação física, mental e social em crianças com CCC nos Estados Unidos (Anjos et al., 2017, Stegemann et al., 2019, Ulkowski et al., 2019).

A musicoterapia foi integrada a Política Nacional de Práticas Integrativas e Complementares (PNPIC) no SUS, por meio da portaria $\mathrm{N}^{\circ} 849$, de 27 de março de 2017. Objetiva desenvolver potenciais e restabelecer funções do indivíduo, favorecendo o desenvolvimento criativo, emocional e afetivo e, fisicamente, ativa o tato e a audição, a respiração, a circulação e os reflexos. Dessa forma, pode ser utilizada como um recurso de cuidado junto a outras práticas, facilitando abordagens interdisciplinares, pois promove relaxamento, conforto e prazer no convívio social, facilitando o diálogo entre os indivíduos e profissionais (Brasil, 2017).

Um estudo de revisão de literatura apontou que a musicoterapia também pode ser aplicada em crianças, como modalidade alternativa de intervenção. No entanto, há escassez de estudos recentes relacionados à temática, o que corrobora a posição de que esta deva ser mais difundida e pesquisada no Brasil (Anjos et al, 2017).

Desse modo, este artigo tem como objetivo descrever os efeitos da musicoterapia aplicada às crianças com CCC sob a ótica do/a familiar.

\section{Metodologia}

Pesquisa de natureza interventiva e participativa, cujas raízes epistemológicas estão nas ciências sociais e na necessidade de responder questões de pesquisa aplicadas. Parte da premissa de que é preciso "transformar para conhecer", ressignificando o papel do pesquisador de alguém que analisa a realidade para alguém que pode transformá-la (Teixeira \& Megid, 2017).

Nesse sentido, o pesquisador assume um papel de responsabilidade social e política na transformação de uma realidade, interferindo por meio de ações específicas e observação do seu impacto na vida dos participantes. A relação entre participantes e pesquisadores é dinâmica e se constrói durante o processo de pesquisa, sendo esse vínculo ou falta dele parte da produção de dados (Teixeira \& Megid, 2017). 


\section{Intervenções}

De março a abril de 2021, foram realizadas de três a quatro sessões individuais de musicoterapia com cada criança, totalizando 43 intervenções. Cada intervenção foi realizada semanalmente pelo pesquisador, sob supervisão de um educador artístico especialista em musicoterapia e atuante na área há mais de dez anos.

Com um violão acústico semiflat modelo SN95 com encordoamento de aço, as sessões foram mediadas pela música popular brasileira e tiveram duração média de 37 minutos, sendo a mais curta de 25 minutos e a mais longa de 50 minutos. $\mathrm{O}$ encerramento foi determinado pela criança e por seu familiar.

Durante as sessões, as crianças eram estimuladas a se movimentar conforme o ritmo da música, sendo acompanhadas pelo fisioterapeuta responsável por seu tratamento neuromuscular.

\section{Procedimentos de produção de dados}

Ao final de cada intervenção, anotações de campo com observações acerca da prática interventiva eram registradas pelo pesquisador. Essas anotações compuseram diários de campo semanais que foram utilizados como medida de modulação dos processos de produção e análise de dados. Essa estratégia permitiu a reflexão teórica e crítica do processo empírico, a partir da construção de uma narrativa ponderada acerca de sua experiência no campo (Kroeff et al., 2020).

Entrevistas semiestruturadas, as quais permitiram focar nos efeitos após as intervenções, foram realizadas com os familiares das crianças ao final delas. Essas foram orientadas por questões abertas que exploravam o comportamento cotidiano de vida da criança após a prática de musicoterapia (DeJonckheere, Vaughn, 2019). Todas foram gravadas com gravador digital.

\section{Análise de dados}

Os dados foram submetidos à análise de conteúdo indutivo, cujos procedimentos envolvem 1) preparo dos dados de acordo com formatação e inteligibilidade do texto; 2) leitura detalhada do material e consideração de diversos significados inerentes ao texto; e 3) identificação de similaridade e recorrência para codificação de categorias (Kyngäs, 2020).

Nesta pesquisa, a primeira etapa foi realizada com o auxílio de quadros analíticos organizados em três colunas: código do/a participante; corpus textual da entrevista; e observações do diário de campo relevantes ao objeto de estudo. A segunda envolveu o uso de marcação colorimétrica das narrativas que apresentavam algum tipo de similaridade, fazendo perguntas e inferências ao texto durante o processo. Já a terceira consistiu em um processo interpretativo analítico situado no contexto dos participantes de pesquisa, que resultou em duas categorias.

\section{Participantes}

Vinte e três crianças corresponderam à população ativa no ambiente de pesquisa. Desses, dois receberam alta do tratamento, uma não atendia aos critérios de inclusão, uma desistiu e oito não aceitaram participar. Assim, onze crianças foram incluídas no estudo com seus pais (nove mães e dois pais). Os critérios de inclusão foram: crianças de zero a 12 anos de idade com CCC em tratamento presencial no centro de reabilitação, sendo excluídas as que estavam em tratamento domiciliar exclusivo.

Das 11 crianças participantes, a média de idade foi de 4,5 anos, sendo a mais jovem com um ano e a mais velha 12 anos. Elas viviam com as seguintes CCC: transtorno do espectro autista, hidrocefalia, Paralisia Cerebral, Síndrome de Blau, Transtorno do déficit de atenção e hiperatividade (TDAH), Transtorno Opositor Desafiador (TOD), Síndrome de Charge e Deficiência Intelectual, sendo que três crianças possuíam mais de uma dessas condições de saúde simultaneamente. 


\section{Cenário}

A pesquisa foi realizada em um município do interior de Goiás com população estimada de 175 mil habitantes, tendo o perfil epidemiológico marcado pelo trabalho informal e a indústria. $\mathrm{O}$ índice de desenvolvimento humano (IDH) é de $0,76 \mathrm{e}$ 96,8\% das crianças de zero a 14 anos são alfabetizadas (IBGE, 2010, Brasil, 2021).

O cenário das intervenções foi a sala de acompanhamento da fisioterapia em um centro de reabilitação de referência. O centro de reabilitação é um órgão da rede pública de saúde e atende à população do entorno desde 1997. É o único órgão a fornecer atendimento especializado de fisioterapia, ortopedia, fonoaudiologia, psicologia e neurologia no âmbito do SUS na região. Dessa forma, optou-se por não a identificá-la neste artigo, uma vez que isso pode acarretar identificação dos participantes de pesquisa.

\section{Aspectos Éticos}

Este estudo foi executado de acordo com os preceitos da Resolução no 466/12 do Conselho Nacional de Saúde do Ministério da Saúde, que regem as pesquisas incluindo seres humanos. Foi aprovado pelo Comitê de Ética em Pesquisa da instituição proponente em 11 de março de 2021 sob o parecer no 4.586.251 e CAAE: 41238820.1.0000.5595.

As crianças maiores de seis anos de idade assinaram o Termo de Assentimento Livre e Esclarecido por escrito, sendo este revisitado durante todos os procedimentos da pesquisa. Para crianças que apresentavam algum tipo de impedimento motor para ler ou assinar esse documento, obteve-se registro em vídeo ou por meio de impressão digital. A partir dos três anos de idade, as crianças ofereceram assentimento durante as etapas da pesquisa na modalidade oral. Utilizou-se como parâmetro para essa decisão normativas legais e éticas brasileiras e um estudo que determina que as crianças conseguem distinguir conceitos morais de certo e errado a partir dos três anos de idade (Brasil, 1990, Brasil, 2012, Bluebond-Langer, 1980).

Já os familiares cuidadores assinaram o Termo de Consentimento Livre e Esclarecido, sendo respeitado seu direito de desistir em qualquer etapa da pesquisa. Toda documentação referente à pesquisa encontra-se armazenada pelos pesquisadores na modalidade física e digital.

Como o estudo foi realizado durante a pandemia da Covid-19, medidas de distanciamento social de, no mínimo, dois metros, uso de equipamentos de proteção individual, higienização e desinfecção das mãos e material foram implementadas.

\section{Resultados}

As crianças foram diagnosticadas com as CCC até os dois anos de idade, tendo a maior parte de sua vida sob cuidados de terceiros, sejam profissionais de saúde nos serviços que frequentam ou familiares em casa.

Nesse sentido, as narrativas dos familiares tomaram forma a partir de referências adquiridas durante todo o percurso terapêutico da criança, mesmo que esse não tenha sido o objeto de investigação. Nas entrevistas, eles descreveram suas impressões e experiências acerca dos comportamentos das crianças durante e após as sessões de musicoterapia. Os familiares inferiram conexões e relações entre o comportamento da criança e as sessões realizadas durante o estudo. Emergiram duas categorias: Mudança de Comportamento ao Longo das Sessões e Mudanças de Comportamentos em Casa.

\section{Mudança de Comportamento ao Longo das Sessões}

Os familiares relataram que perceberam mudança de comportamento da criança no decorrer das sessões. A primeira sessão foi marcada pelo medo e agitação do encontro com um novo profissional, um desconhecido cujo vínculo ainda não havia se estabelecido.

Na primeira sessão de musicoterapia, ela estava muito travada, só olhando e com medo. Ela sempre teve muito medo 


\section{de profissionais diferentes. (F1)}

Meu filho, na primeira sessão, foi [...] um pouco resistente porque não conhecia as pessoas que estavam fazendo a intervenção. $(\mathrm{F} 3)$

C6 era muito agitado, quebrava tudo, eu não tinha muito noção das coisas. Ele adora música, mas batia em tudo no início. (F6)

Na segunda sessão, observou-se aumento de foco nas crianças com autismo severo. Antes demonstravam agitação e, à medida que estabeleceram vínculo com pesquisador, passaram a se interessar pelo instrumento musical. Isso foi ressignificado como uma forma de promover calma e tranquilidade, controlando sua raiva.

Já na segunda sessão a gente percebeu que quando começava a tocar o violão, quando começava a música, ele parava tudo e ficava ali e prestava a atenção. Então eu pude perceber que é uma coisa que ele se interessa muito que é a música. (F3)

Depois da musicoterapia, ele conversava, a gente via ele mais calmo, tentando controlar a raiva dele. (F6)

As sessões passaram a ser integradas ao atendimento do fisioterapeuta de confiança da criança, cujas implicações musculoesqueléticas envolviam rigidez muscular. Assim, a música era tocada enquanto procedimentos de alongamento e relaxamento eram realizados de forma concomitante. Durante essas sessões, os familiares associaram a musicoterapia com a melhora no manuseio da criança durante os exercícios e o aumento da flexibilidade muscular, devido ao relaxamento proporcionado pela música.

Com a música, os profissionais conseguiram manuseá-la para fazer a fisioterapia de uma forma mais tranquila. (F1)

Com a musicoterapia, eu notei que ele está com a musculatura menos atrofiada e mais flexível. Ele relaxa mais. (F11) Ele ficou mais calmo depois que começaram as atividades. (F8)

Ele está mais calmo durante a fisioterapia (F10)

A redução do medo dos profissionais diferentes, aumento do foco, estabelecimento de vínculo e relaxamento muscular nos momentos de alongamento relatados pelos familiares também foram registrados nos diários de campo. Tais mudanças foram consideradas significativas para o acompanhamento da criança durante a sessão de musicoterapia.

\section{Mudanças de Comportamento em Casa}

Nove familiares observaram mudanças no comportamento das crianças, quando foram submetidos à musicoterapia. $\mathrm{O}$ contato com a música proporcionou melhora na comunicação e na socialização com a família. Três crianças foram descritas como brincalhonas, comunicativas, menos irritadas e com maior facilidade de interação com pessoas.

Em casa um pouco mais brincalhão, comunicativo. (F2)

Com a musicoterapia, a gente percebeu que ele ficou bem mais fácil de interagir com as pessoas. (F3)

Agora ele está sereno, já chega pronto para brincar, não grita mais. (F7)

Depois da musicoterapia, ele melhorou o comportamento, já deu os primeiros passos sozinho (F5)

No que tange o desenvolvimento da criança, dois familiares imprimiram articulação da musicoterapia com melhora da fala, relatando que as crianças passaram a construir frases com sentido e com maior número de palavras. Esse tipo de resultado pode ter ocorrido devido à característica estimuladora da musicoterapia associada às intervenções de cuidado implementadas 
pela equipe multiprofissional, como o brincar, exercícios na musculatura mandibular e orientação dos familiares para conversarem e estimularem a criança a se comunicar.

Desenvolveu a fala dela, ela se comunica melhor. Aumentou à concentração, melhorou a dicção das palavras. (F6) Antes ele não falava coisa com coisa e hoje o que ele fala tem sentido, ele realmente está se comunicando melhor. (F7)

Quatro familiares realizaram associação empírica da mudança de comportamento das crianças, quando exposta à música. Por meio da observação, acreditam que a música promove relaxamento e deixa a criança mais tranquila, especialmente no momento de manuseio durante os cuidados do dia a dia (higiene, mudança de posição, troca de fraldas e curativos). A partir disso, um familiar passou a implementar a estratégia da musicoterapia em casa.

A musicoterapia tem mudado, ela está mais tranquila. (F11)

[...] mais depois com a música se tornou uma menina mais calma. (F9)

Ele ficou bem mais calmo, bem mais tranquilo, então eu percebi a melhora no comportamento dele após a sessão de musicoterapia. (F3)

Em casa, notei que ela (criança) associava (manuseio) quando ela ouvia uma música. Colocava música e ela já ficava mais tranquila, deixava que a gente mexesse nela sem brigar, uma coisa que, antes, ela não deixava. (F1)

Foi reconhecido que a musicoterapia contribuiu para que a criança atingisse marcos do desenvolvimento com maior rapidez. Por meio do estímulo sonoro e sensorial, percebeu-se que as crianças se encontravam mais atentas e interativas com o ambiente domiciliar.

Ele aprendeu a pular, cantar e dançar. Ele era mais paradinho e, agora, noto a diferença quando coloco música. (F1)

Ele está bem mais esperto, atento a movimentos. (F2)

[...] fazer musicoterapia a gente observa que ele fica mais atento, presta mais atenção. (F8)

Depois de iniciar a musicoterapia e agregar com a fisioterapia, conseguiu dar os primeiros passos, coisa que não fazia antes mesmo de fazer os tratamentos. (F4)

Em casa, os familiares observaram mudanças positivas no comportamento da criança após as sessões de musicoterapia, descrevendo a criança como mais brincalhona, comunicativa, interativa, concentrada e tranquila. Isso denota que as sessões de musicoterapia tiveram bons resultados também no ambiente familiar, onde os familiares passaram a utilizar a música para auxiliar na realização de atividades cotidianas com a criança.

\section{Discussão}

Para os familiares cuidadores de crianças com CCC, os efeitos da musicoterapia emergiram no plano comportamental durante as sessões e em casa. Durante as sessões, observou-se o aumento do vínculo por meio da interação musical; a associação da prática com diminuição do medo e da agressividade; aumento do foco e da tranquilidade. Esses resultados apresentados por meio de dados qualitativos corroboram com informações apontadas por estudos clínicos no campo da psicologia interpessoal (Fachner et al., 2019, Dvir et al., 2020, Samadi et al., 2018).

A intervenção musical no cuidado de enfermagem pode ser realizada de diversas maneiras, utilizando músicas prédeterminadas, solicitações das próprias crianças e instrumentos musicais, manuseados pela equipe ou pelas crianças. Essa interação colabora para a expressão e comunicação da criança de modo que ela passa a confiar no profissional que lhe presta o 
cuidado em saúde. Dessa forma, a partir do relacionamento de confiança estabelecido, os familiares ficam mais tranquilos contribuindo para que os procedimentos sejam realizados com maior facilidade (Rodrigues et al., 2018).

A sincronia interpessoal demonstra que fisiologicamente funções neurológicas são ativadas durante o processo de musicoterapia e podem ocasionar relaxamento muscular. Essas funções ocorrem de maneira sincronizada entre a criança e o musicoterapeuta e parecem influenciar no estabelecimento de vínculo (Fachner et al., 2019, Dvir et al., 2020). Sessões de quinze minutos de musicoterapia podem impactar na sincronia intercerebral da criança com CCC e seu responsável e, consequentemente, as relações estabelecidas entre eles (Samadi et al., 2021).

No campo da psicologia, aponta-se a musicoterapia como uma atividade lúdica com potencial para manejo de sentimentos como ansiedade e medo associada ao estresse em crianças. Como recurso terapêutico não farmacológico, favorece o desenvolvimento da criatividade, sociabilidade e comunicação, reduzindo a dor e aumentando a aceitação nos procedimentos invasivos nos ambientes de saúde (Anjos et al., 2017).

Pesquisadores evidenciaram que o uso de música em fones de ouvido durante intervenções dolorosas pode ser uma medida segura, fácil e de baixo custo para diminuição de sentimentos de dor e medo, quando comparados ao grupo de crianças que não receberam essa intervenção (Thanh et al., 2010).

$\mathrm{Na}$ Austrália, estudos registram o impacto dessa prática terapêutica enquanto recurso lúdico para aumento do vínculo, sociabilidade, sentimentos positivos e maior qualidade no tempo passado entre crianças e pais, reforçando a importância do trabalho colaborativo dos profissionais da área da saúde com eles (Thompson \& McFerran, 2015, Thompson et al., 2018). O desenvolvimento das visitas musicais nos hospitais possibilita benefícios para a criança e os familiares, tais como sentimentos de tranquilidade, paz, alegria e calma (Oliveira \& Cardoso, 2014).

Nesse sentido, os familiares são expostos a diversos tipos de cuidados e conhecimentos, podendo apreendê-los durante o período de internação da criança para utilizá-los em casa (Macedo et al., 2017, Góes \& Cabral, 2017). No cenário domiciliar, a incorporação da música no cotidiano de uma das crianças desta pesquisa aumentou o desenvolvimento da fala e melhorou a comunicação. A utilização da musicoterapia em casa proporciona que a criança seja assistida de forma integral e resolutiva, uma vez que o cuidado transcende a esfera fisiológica e adentra a esfera emocional e relacional (Yang, 2016).

Durante a pandemia da Covid-19, a aplicação da musicoterapia no ambiente domiciliar evidenciou a diminuição do estresse provocado pelo distanciamento social nas crianças que apresentavam CCC com implicações de desenvolvimento. O uso de aplicativos de comunicação e o acesso à internet contribuíram para que essas crianças permanecessem no programa por meio da telessaúde e continuassem aderentes aos seus tratamentos (Bompard et al., 2021).

Um estudo de revisão de literatura apontou que a musicoterapia influencia positivamente as crianças no que se refere a socialização, interação, comunicação, psicomotricidade e linguagem. Durante a utilização da musicoterapia a criança interage com sua família, colegas, cuidadores e terapeutas, melhorando seu humor, atenção e envolvimento nas atividades (Oliveira et al., 2021).

Outro estudo apontou as mudanças que a musicoterapia possibilitou no desenvolvimento da fala e a melhora da relação da criança com a família. O ritmo e as melodias proporcionadas pelas sessões desse tipo de terapia podem aumentar o interesse da criança possibilitando que portas de comunicação sejam abertas (Samadi et al., 2021, Moreira \& Freitas, 2016). Assim, a música enquanto recurso terapêutico, lúdico, não farmacológico e de baixo custo pode ser utilizado pela enfermagem para contribuir com a promoção do cuidado integral às crianças em diversos cenários de saúde e em casa.

A Enfermagem, enquanto campo de conhecimento, pode articular o uso de práticas de cuidado de musicoterapia no Processo de Enfermagem às crianças com CCC. Essa articulação pode suscitar a incorporação de um novo profissional na equipe de saúde, o musicoterapeuta, oferecendo transdisciplinaridade no cuidado.

Conhecer o impacto da musicoterapia no cuidado às crianças com CCC fornece material científico para que a 
Enfermagem amplie o campo de aplicação de abordagens que favoreçam a atenção integral a essa população. Os resultados deste estudo contribuem para que o Enfermeiro engaje em um processo reflexivo para prescrever cuidados alternativos a essas crianças, com abordagem multiprofissional que transcende os profissionais tradicionalmente da saúde no cuidado que contribua com a promoção da saúde coerente com a política de humanização do SUS.

O caráter relaxante e tranquilizador da música foi observado no serviço e em casa, sendo implementado no cotidiano de cuidado de uma participante. Esses resultados corroboram achados de estudos quantitativos e qualitativos realizados no Brasil e exterior. No entanto, é necessário investir em estudos que descrevam, analisem e avaliem a possibilidade de implementação da musicoterapia nos serviços.

\section{Considerações Finais}

De acordo com o familiar, a inserção da prática de musicoterapia no cotidiano de cuidado de crianças com CCC impactou positivamente suas vidas no serviço de saúde e em casa. No serviço de saúde, identificou-se que, ao longo das sessões, houve o aumento do vínculo, foco, relaxamento e flexibilidade motora-muscular. Em casa, o relacionamento da criança com seus familiares melhorou. $\mathrm{O}$ aumento da forma como interage foi associado com a musicoterapia, uma vez que a criança recebeu mais estímulos que antes do início da prática.

Como limite do estudo, tem-se a dificuldade de acesso aos participantes, visto a situação da pandemia e pela inexistência de cenários específicos para o atendimento dessas crianças no município de realização da pesquisa. A pesquisa visa contribuir na ratificação da importância de ampliar estudos sobre musicoterapia e suas implicações à qualidade de vida das crianças com CCHC, tendo a colaboração entre criança, familiares e profissionais de diversas áreas de conhecimento.

\section{Referências}

Banerjee, T., Khan, A., \& Kesavan, P. (2021). Impact of lockdown and school closure on children in special schools: a single-centre survey. BMJ Paediatrics Open, 5(1), e000981. https://doi.org/10.1136/bmjpo-2020-000981

Bluebond-Langner, M. (1980). The Private Worlds of Dying Children. Princeton University Press, 1980.

Bompard, S., Liuzzi, T., Staccioli, S., D’Arienzo, F., Khosravi, S., Giuliani, R., \& Castelli, E. (2021). Home-based music therapy for children with developmental disorders during the COVID-19 pandemic. Journal of Telemedicine and Telecare, 1357633 X2098121. https://doi.org/10.1177/1357633X20981213

Lei no 8.069/1990. Dispõe sobre o Estatuto da Criança e do Adolescente e dá outras providências., Diário Oficial da União - Presidência da República (1990). http://www.planalto.gov.br/ccivil_03/leis/18069.htm

Portaria no 849/2017. Inclui a Arteterapia, Ayurveda, Biodança, Dança Circular, Meditação, Musicoterapia, Naturopatia, Osteopatia, Quiropraxia, Reflexoterapia, Reiki, Shantala, Terapia Comunitária Integrativa e Yoga à Política Nacional de Práticas Integrativas e Complementares., Ministério da Saúde, Gabinete do Ministro - Diário Oficial da União (2017). https://bvsms.saude.gov.br/bvs/saudelegis/gm/2017/prt0849_28_03_2017.html

Brasil. (2018). Política Nacional de Atenção Integral à Saúde da Criança: orientações para implementação. Ministério da Saúde. Editora MS - OS 2018/0048. https://portaldeboaspraticas.iff.fiocruz.br/wp-content/uploads/2018/07/Política-Nacional-de-Atenção-Integral-à-Saúde-da-Criança-PNAISC-Versão-

Eletrônica.pdf

Clemens, V., Deschamps, P., Fegert, J. M., Anagnostopoulos, D., Bailey, S., Doyle, M., Eliez, S., Hansen, A. S., Hebebrand, J., Hillegers, M., Jacobs, B., Karwautz, A., Kiss, E., Kotsis, K., Kumperscak, H. G., Pejovic-Milovancevic, M., Christensen, A. M. R., Raynaud, J.-P., Westerinen, H., \& VisnapuuBernadt, P. (2020). Potential effects of "social" distancing measures and school lockdown on child and adolescent mental health. European Child \& Adolescent Psychiatry, 29(6), 739-742. https://doi.org/10.1007/s00787-020-01549-w

DeJonckheere, M., \& Vaughn, L. M. (2019). Semistructured interviewing in primary care research: a balance of relationship and rigour. Family Medicine and Community Health, 7(2), e000057. https://doi.org/10.1136/fmch-2018-000057

Dvir, T., Lotan, N., Viderman, R., \& Elefant, C. (2020). The body communicates: Movement synchrony during music therapy with children diagnosed with ASD. The Arts in Psychotherapy, 69, 101658. https://doi.org/10.1016/j.aip.2020.101658

Elmac1, D., \& Cevizci, S. (2015). Dog-Assisted Therapies and Activities in Rehabilitation of Children with Cerebral Palsy and Physical and Mental Disabilities. International Journal of Environmental Research and Public Health, 12(5), 5046-5060. https://doi.org/10.3390/ijerph120505046

Fachner, J. C., Maidhof, C., Grocke, D., Nygaard Pedersen, I., Trondalen, G., Tucek, G., \& Bonde, L. O. (2019). "Telling me not to worry...” Hyperscanning and Neural Dynamics of Emotion Processing During Guided Imagery and Music. Frontiers in Psychology, 10. https://doi.org/10.3389/fpsyg.2019.01561 
Fegert, J. M., Vitiello, B., Plener, P. L., \& Clemens, V. (2020). Challenges and burden of the Coronavirus 2019 (COVID-19) pandemic for child and adolescent mental health: a narrative review to highlight clinical and research needs in the acute phase and the long return to normality. Child and Adolescent Psychiatry and Mental Health, 14(1), 20. https://doi.org/10.1186/s13034-020-00329-3

Góes, F. G. B., \& Cabral, I. E. (2017). Discursos sobre cuidados na alta de crianças com necessidades especiais de saúde. Revista Brasileira de Enfermagem, 70(1), 163-171. https://doi.org/10.1590/0034-7167-2016-0248

Gonzaga Dos Anjos, A., Daniel Montanhaur, C., Bruno, É., Campos, V., Luiza, A., Pereira, R., Piovezana, D., Santos Montalvão, J., Maria, C., \& Neme, B. (2017). Musicoterapia como estratégia de intervenção psicológica com crianças: uma revisão da literatura. Gerais : Revista Interinstitucional de Psicologia, 10(2), 228-238. http://pepsic.bvsalud.org/scielo.php?script=sci_arttext\&pid=S1983-82202017000200008\&lng=pt\&nrm=iso\&tlng=pt

Graham, H. K., Rosenbaum, P., Paneth, N., Dan, B., Lin, J.-P., Damiano, D. L., Becher, J. G., Gaebler-Spira, D., Colver, A., Reddihough, D. S., Crompton, K. E., \& Lieber, R. L. (2016). Cerebral palsy. Nature Reviews Disease Primers, 2(1), 15082. https://doi.org/10.1038/nrdp.2015.82

Herdy, A. M., \& Carmo, C. de F. do. (2016). Os efeitos da musicoterapia em pacientes portadores do transtorno do espectro autista. Revista Interdisciplinar Pensamento Científico, 2(2). https://doi.org/10.20951/2446-6778/v2n2a17

IBGE. (2010). Pesquisa nacional por amostra de domicílios. Um panorama da saúde no Brasil. Acesso e utilização dos serviços, condições de saúde e fatores $\mathrm{de}$ risco e proteção à saúde 2008. In Instituto Brasileiro de Geografia e Estatística. http://bvsms.saude.gov.br/bvs/publicacoes/pnad_panorama_saude_brasil.pdf

Kroef, R. F. da S., Gavillon, P. Q., \& Ramm, L. V. (2020). Diário de Campo e a Relação do(a) Pesquisador(a) com o Campo-Tema na Pesquisa-Intervenção. Estudos e Pesquisas Em Psicologia, 20(2), 464-480. https://doi.org/10.12957/epp.2020.52579

Kyngäs, H. (2020). Inductive Content Analysis. In The Application of Content Analysis in Nursing Science Research (pp. 13-21). Springer International Publishing. https://doi.org/10.1007/978-3-030-30199-6_2

Macedo, I. F. de, Souza, T. V. de, Oliveira, I. C. dos S., Cibreiros, S. A., Morais, R. de C. M. de, \& Vieira, R. F. C. (2017). Nursing team's conceptions about the families of hospitalized children. Revista Brasileira de Enfermagem, 70(5), 904-911. https://doi.org/10.1590/0034-7167-2016-0233

Moreira, M. C. N., Albernaz, L. V., Sá, M. R. C. de, Correia, R. F., \& Tanabe, R. F. (2017). Recomendações para uma linha de cuidados para crianças e adolescentes com condições crônicas complexas de saúde. Cadernos de Saúde Pública, 33(11). https://doi.org/10.1590/0102-311x00189516

Moura, E. C. de, Moreira, M. C. N., Menezes, L. A., Ferreira, I. A., \& Gomes, R. (2017). Complex chronic conditions in children and adolescents: hospitalizations in Brazil, 2013. Ciência \& Saúde Coletiva, 22(8), 2727-2734. https://doi.org/10.1590/1413-81232017228.01992016

Nguyen, T. N., Nilsson, S., Hellström, A.-L., \& Bengtson, A. (2010). Music Therapy to Reduce Pain and Anxiety in Children With Cancer Undergoing Lumbar Puncture: A Randomized Clinical Trial. Journal of Pediatric Oncology Nursing, 27(3), 146-155. https://doi.org/10.1177/1043454209355983

Novak, I., \& Honan, I. (2019). Effectiveness of paediatric occupational therapy for children with disabilities: A systematic review. Australian Occupational Therapy Journal, 66(3), 258-273. https://doi.org/10.1111/1440-1630.12573

Oliveira, F. de, Oliveira, F. V. de, Neta, M. M. R., Magalhães, J. M., Oliveira, A. D. da S., Amorim, F. C. M., \& Carvalho, C. M. S. de. (2021). Contribution of music therapy to autism spectrum disorder: an integrative literature review. Journal of Nursing and Health, 11(1). https://doi.org/10.15210/jonah.v11i1.17779

Oliveira, L. N. de, \& Cardoso, C. P. (2014). Cuidados de enfermagem à criança hospitalizada: efeitos da música como terapêutica complementar no cuidar em pediatria. Brazilian Journal of Music Therapy. https://musicoterapia.revistademusicoterapia.mus.br/index.php/rbmt/article/view/215

Rodrigues, F. M., Palma, L. C. de O. S., Silva, J. de O. e, Santos, A. P. de O., Neta, A. I. de O., Fonseca, A. D. G., \& Silva, C. S. de O. e. (2018). Hospitalização infantil: influência da brinquedoterapia e da musicoterapia na assistência à criança. Revista Eletrônica Acervo Saúde, (10), S1123-S1128. https://doi.org/10.25248/REAS183_2018

Samadani, A., Kim, S., Moon, J., Kang, K., \& Chau, T. (2021). Neurophysiological Synchrony Between Children With Severe Physical Disabilities and Their Parents During Music Therapy. Frontiers in Neuroscience, 15. https://doi.org/10.3389/fnins.2021.531915

Stegemann, T., Geretsegger, M., Phan Quoc, E., Riedl, H., \& Smetana, M. (2019). Music Therapy and Other Music-Based Interventions in Pediatric Health Care: An Overview. Medicines, 6(1), 25. https://doi.org/10.3390/medicines6010025

Teixeira, P. M. M., \& Megid Neto, J. (2017). Uma proposta de tipologia para pesquisas de natureza interventiva. Ciência \& Educação (Bauru), 23(4), 10551076. https://doi.org/10.1590/1516-731320170040013

Thompson, G. A., Shanahan, E. C., \& Gordon, I. (2019). The role of music-based parent-child play activities in supporting social engagement with children on the autism spectrum: A content analysis of parent interviews. Nordic Journal of Music Therapy, 28(2), 108-130. https://doi.org/10.1080/08098131.2018.1509107

Thompson, G., \& McFerran, K. S. (2015). "We've got a special connection": qualitative analysis of descriptions of change in the parent-child relationship by mothers of young children with autism spectrum disorder. Nordic Journal of Music Therapy, 24(1), 3-26. https://doi.org/10.1080/08098131.2013.858762

Ulkowski, I. D. P. I., Cunha, R. R. dos S., \& Pinheiro, N. N. B. (2020). Da musicoterapia à musicoterapia orientada pela teoria psicanalítica: fundamentos epistemológicos. Revista InCantare, 10(1), 1-166. http://periodicos.unespar.edu.br/index.php/incantare/article/view/3151

UNICEF. (2003). The State of the World's Children 2004. The United Nations Children's Fund.

Yang, Y.-H. (2016). Parents and Young Children with Disabilities: The Effects of a Home-Based Music Therapy Program on Parent-Child Interactions. Journal of Music Therapy, 53(1), 27-54. https://doi.org/10.1093/jmt/thv018. 